\title{
Highly Sensitive and Robust Capillary Electrophoresis-Electrospray lonization-Mass Spectrometry: Interfaces, Preconcentration Techniques and Applications
}

https://doi.org/10.1515/revac-2020-0112

Received March 03, 2020; accepted June 16, 2020

\begin{abstract}
Capillary electrophoresis (CE) coupled with mass spectrometry (MS) through electrospray ionization (ESI) is a promising alternative technique to liquid chromatography-ESI-MS (LC-ESI-MS) due to its high separation efficiency and high detection sensitivity. A sensitive and robust interface is essential in CE-ESI-MS. Continued development of CE-ESI-MS interfaces in the last decade, including junction-at-the-tip interfaces and sheathless interfaces, has improved the sensitivity and robustness of CE-ESI-MS significantly. The limited loading capacity of $\mathrm{CE}$, one of major reasons that limits the utility of $\mathrm{CE}$ as a routine separation method, has also been addressed effectively by the use of in-capillary sample preconcentration techniques, such as transient CITP/CZE and dynamic $\mathrm{pH}$ junction. CE-ESI-MS could yield higher sensitivity as compared with the conventional LC-ESI-MS, and, therefore, is capable of identifying more proteins and peptides when the sample amount is very limited, such as single cell analysis. To improve the protein sequence coverage, CE-ESI-MS can also be used as a complementary technique to LC-ESI-MS, or combined with reversed phase LC to form a two dimensional separation technique. CE-ESI-MS is also effective in quantifying targeted peptides/proteins in complex bio-matrix.
\end{abstract}

Keywords: CE-ESI-MS, interface, samplepreconcentration technique, sensitive, robust

\footnotetext{
*Corresponding author: tangkeqi@nbu.edu.cn

${ }^{1}$ Faculty of Electrical Engineering and Computer Science, Ningbo University, Ningbo 315211, P. R. China

${ }^{2}$ Zhejiang Provincial Key Laboratory of Advanced Mass Spectrometry and Molecular Analysis, Institute of Mass Spectrometry, Ningbo University, Ningbo 315211, P. R. China
}

\section{Introduction}

Identification and quantification of trace amounts of analytes in complex bio-matrix is a highly desirable but challenging task, as these minute analytes, such as many important disease related biomarkers in blood plasma, often exist at very low concentrations and are heavily 'masked' by many nonsignificant species with more than 10 orders of magnitude higher concentrations [1]. In order to effectively detect these ultra-low abundance analytes among the endogenous high abundance interfering species, a combination of sample separation method with mass spectrometry (MS) is essential for a high-sensitivity and selectivity analysis. Although the resolution of gas or liquidphase based separations are relatively lower as compared to MS, they both provide an effective way to simplify the sample mixture and alleviate the ion suppression effect during the ionization step prior to the MS analysis [2].

Capillary electrophoresis (CE) is considered as a promising alternative separation technique to liquid chromatography (LC), due to its high separation efficiency, broad sample compatibility, relatively short analysis time and low sample consumption. The separation mechanism of $\mathrm{CE}$ is based on charge-to-size ratio differences of analytes, which is different from the LC separation mechanism. Combining LC with CE separation can often provide complementary information on the composition of a complex biological sample.

Coupling CE to MS through electrospray ionization (ESI) was a major step forward in the development and application of capillary electrophoresis, as first reported in 1987 [3]. More than two decades later, ESI still remains the primary ionization technique in CE-MS. With ESI, the separated analytes in CE at low flow rates can be efficiently transferred from the liquid phase into gas-phase ions. ESI is also a well-known 'soft ionization' technique and is particularly adaptable in producing multiply charged ions from macromolecules such as proteins or peptides. 
The challenge in CE-ESI-MS is the effective coupling of CE with ESI in order to achieve high MS detection sensitivity while preserving high CE separation efficiency. This requires a robust CE-ESI-MS interface that allows generation of a stable and high efficiency electrospray with minimal separation band-broadening. The robustness of a CE-ESI-MS interface generally means stable electrospray, good reproducibility and extensive longevity, which are necessary for CE-MS to be widely adopted in practical applications. A number of comprehensive reviews about the development and applications of CE-MS, including both CE and Microchip capillary electrophoresis (MCE) coupling to MS through ESI, have been already published [4-7]. Noticeably, a microchip-based CE cartridge was recently developed by the Ramsey group to achieve high efficiency coupling of CE with MS [8, 9]. This so called ZipChip has been recently commercialized by 908 devices (Boston, MA). Recently, the dramatic improvement in the CE-MS interface and preconcentration techniques has made CE-MS an important analytical tool for large scale topdown and bottom-up proteomics [10-12]. Nearly 6000 proteoforms from the Escherichia coli proteome could be identified by advanced CE-MS, which is one of the largest top down proteomic datasets so far [13]. 8200 protein groups and 70,000 unique peptides could be identified by coupling CE-MS to SCX-RPLC for deep bottom-up proteomic [14]. This article focused on the theme of highly sensitive and robust CE-MS with special attention to the latest developments in the interface and preconcentration techniques and related applications. The development of highly sensitive and robust CE-ESI-MS interfaces will be first introduced with a detailed discussion of the pros and cons of various interfaces (section 2), followed by a detailed discussion of the development effort to overcome limited sample loading capacity of CE (section 3), one of the major reasons that limits the utility of $\mathrm{CE}$ as a routine separation method. Some examples of the recent applications in using highly sensitive and robust CE-ESI-MS will then be presented (section 4) followed by the major conclusions of the chapter (section 5).

\section{CE-ESI-MS Interfaces}

In CE-ESI-MS, samples in background electrolyte (BGE) are separated according to their charge-to-size ratios or electric mobilities in a strong electric field formed by a large potential difference applied at both ends of the separation capillary. The separated sample analytes are then ionized to form gas-phase ions by electrospray and introduced into MS for detection based on their massto-charge ratios. To make seamless integration of the CE and ESI processes, a well designed CE-ESI-MS interface is critical to preserve the CE separation quality and achieve high MS sensitivity. The primary concern here is how to add an additional high voltage into the separation flow stream to allow independent control of the electrospray operation without causing sample dilution or band broadening in CE separation. As illustrated in Figure 1, coupling CE to MS through ESI can be considered as combining two closed electric circuits, including a CE circuit and an ESI-MS circuit, which share the same electrode in this three-electrode system consisting of CE inlet, CE exit/ESI emitter and MS inlet. Tens of kilovolts potential for a meter long separation capillary and several kilovolts potential are typically required to maintain a high efficient CE separation and a stable ESI operation.

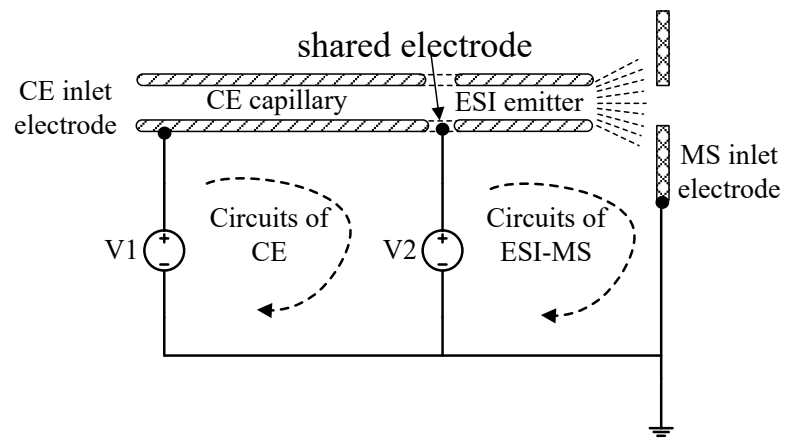

Figure 1 Electrical perspective of a CE-ESI-MS interface

To obtain high CE separation quality, stable electrospray, and high MS detection sensitivity, a robust electrical contact at the shared electrode is critical but challenging in CE-ESI-MS interfaces design. Base on the shared electrode design, all the major CE-ESI-MS interfaces developed so far can be classified into two different categories, namely a sheath liquid interface and a sheathless interface. In a sheath liquid interface, conductive sheath liquid is used to form a liquid junction with the CE separation liquid at the exit of the separation capillary/ESI emitter. ESI voltage is applied to the sheath liquid to close the CE-ESI-MS circuit. In a sheathless interface, ESI voltage is directly applied to the separation liquid at the exit of the separation capillary either via a conductive surface of the ESI emitter capillary or via a conductive liquid as described in detail below.

\subsection{Sheath liquid interfaces}

Coaxial sheath liquid CE-ESI-MS interface was first developed in early 1988 [15]. In this interface 
configuration, the separation capillary was inserted into two larger diameter tubes coaxially (Figure 2). The shared electrical contact of CE and ESI was achieved by applying a voltage on the first metal tube, through which a conductive sheath liquid was delivered and merged with CE effluent at the exit of separation capillary. The second tube was filled with nebulizing gas to aid the ESI process. The performance of coaxial sheath liquid interface was found to be quite robust, and the use of electrospray friendly sheath liquid also allowed the flexibility of using a wide range of BGE solvents for CE separation. The sheath liquid flow rate was typically in the range of 1 to $10 \mu \mathrm{L} / \mathrm{min}$, while the operating flow rate in CE was usually between 20 and $100 \mathrm{~nL} / \mathrm{min}$. The much higher sheath liquid flow rate would significantly dilute the concentration of analyte eluting from the CE separation capillary resulting in a significant loss of overall CE-ESI-MS sensitivity.

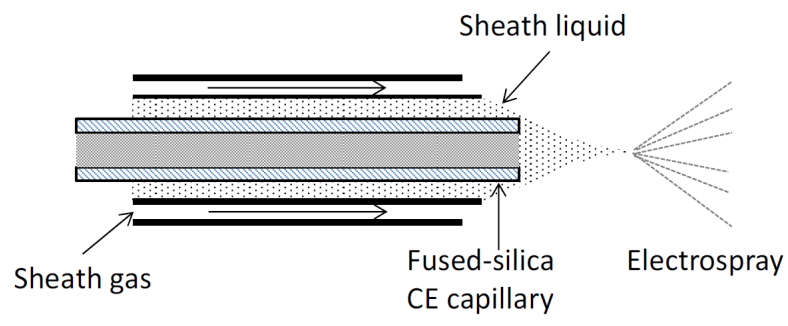

Figure 2 Schematic of the coaxial sheath-liquid interface

To overcome the sample dilution problem in the coaxial sheath liquid interface, liquid junction-at-the-tip interfaces with very low sheath liquid flow rate were proposed to minimize the mixing volume of the sheath liquid flow and CE separated sample flow. An example of the junction-at-the-tip interface was developed by Chen's group [16]. In this interface, the terminal end of the separation capillary was inserted into a beveled stainless steel ESI emitter. Conductive sheath liquid was delivered to the stainless steel emitter by using a tee junction. The very small mixing volume between the terminal end of CE separation capillary and the tip of the ESI emitter allowed the use of sheath liquid flow rate as low as $0.1 \mu \mathrm{L} / \mathrm{min}$, which was at least 10 -fold less than that used in the initial coaxial sheath-liquid interface. This relieved the sample dilution in CE-ESI-MS interface significantly. Compared with a commercial sheath-flow CE-MS interface operating under optimized conditions, limit of detection (LOD) of amino acids was improved 5-fold on average by using this junction-at-the-tip interface.

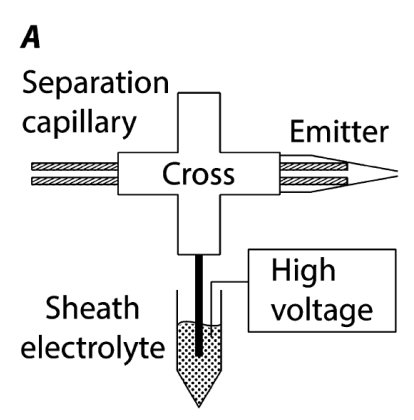

B 1'st generation

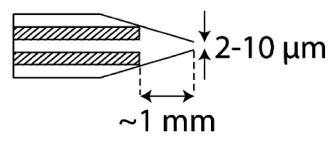

2'nd generation

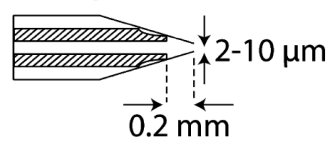

$3^{\prime}$ rd generation

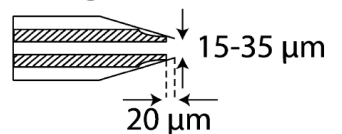

Figure 3 (A) Overall design of the junction-at-the-tip interface proposed in Dovichi's group; (B) Designs of three generations of interface. Reprinted with permission from reference [17]

The design of the junction-at-the-tip interface was perfected by Dovichi's group [17]. Electro-kinetically driven sheath flow was used in their interfaces to further reduce the flow rate of the sheath liquid. Specifically, the separation capillary in these interfaces was threaded through a plastic cross into a glass ESI emitter (Figure 3A). A tube connected a side arm of the cross to a sheath liquid reservoir. When $\sim 1 \mathrm{kV}$ voltage potential was applied to the liquid reservoir, under the effect of electroosmotic flow, the sheath liquid was electro-kinetically pumped at only nanoliters per minute flow rate through the glass emitter, mix with the separated sample, and generate a stable electrospray. Finding an effective way to shorten the distance between the separation capillary distal tip and the emitter orifice was the main focus of the interface development, as simulation results showed that the overall sensitivity of CE-ESI-MS increased as the distance was shortened. After three iterations of the interface development as illustrated in Figure 3B, the distance was reduced ultimately to about $20 \mu \mathrm{m}$. The sample dilution was significantly reduced with the shortening of distance between capillary tip and emitter orifice. Extremely high sensitivity with an estimated detection limit of $\sim 1$ zeptomole was achieved by using this interface coupled with a Q-Exactive mass spectrometer [18].

\subsection{Sheathless interfaces}

Sheathless interface was developed in an attempt to completely eliminate the sample dilution problem. Without the use of sheath liquid in theinterface, the sample flow becomes the only effluent in the electrophoretic 
separation and electrospray processes so that it can be easily controlled at a nanoliter per minute scale to achieve a highly efficient nano flow ESI. These features make the sheathless interface-based CE-ESI-MS potentially more sensitive as compared to sheath liquid interface-based CE-ESI-MS. The major challenge in sheathless interface design is to build a robust sharing electrical contact without the aid of sheath liquid. Various strategies have been proposed to provide the electrical contact, including coating the spray emitter with conductive materials such as gold, silver, copper, nickel, graphite, and conductive polymer, inserting a wire at the emitter tip, and inserting a wire through a hole drilled through the capillary wall. These strategies suffered from one or several common problems including low mechanical robustness, poor reproducibility, short longevity, manufacturing complexity, and unstable electrospray [19]. To overcome these common problems in all coated emitter CE-ESI-MS interfaces, a novel sheathless interface using a chemically etched porous emitter was recently developed with a much improved performance [20]. In this interface design, as shown in Figure 4, [21] a 1 - 1.5 inch long porous surface near the separation capillary exit was created by etching the capillary wall with hydrofluoric acid solution. The capillary with the porous wall was then inserted into a metal tube filled with a conductive liquid. A small portion of the porous wall capillary protrudes about several millimeter beyond the metal tube to serve as an electrospray emitter. The thin porous section of the capillary allows small ions to transport between conductive liquid and solution inside the CE separation capillary to form a robust shared electrical contact. The interface design leaves the capillary inner wall intact and therefore avoids dead volume. Bubble formation due to the electrochemical reaction at high voltage electrode is outside of the separation capillary and therefore does not affect CE separation and electrospray processes. Moreover, the fabrication of porous emitter is reproducible and can be automated, so that this interface design has been commercialized and led to several successful applications as will be highlighted later.

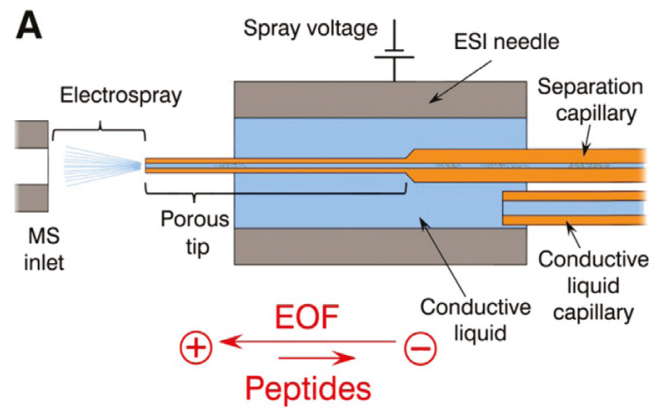

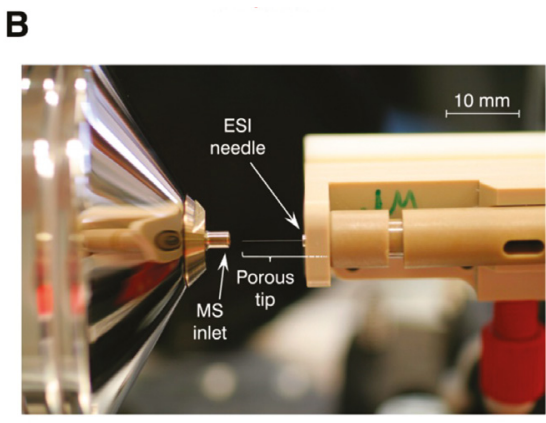

Figure 4 The porous sheathless interface (A) schematic and (B) photograph of the prototype interface. Reprinted with permission from reference [21]

Detailed optimization and performance evaluation of the porous sheathless interface by Busnel and co-workers demonstrated that the interface was capable of generating a stable spray with a wide range of flow rates (below 10 $\mathrm{nL} / \mathrm{min}$ to above $340 \mathrm{~nL} / \mathrm{min}$ ). The capability of this sheathless interface to operate the ESI process at very low flow rate is a very desirable feature as nano-ESI provides advantages of both improving ionization efficiency and reducing ion suppression in the ESI process. Their study also showed that neutral coating on the inner wall of separation capillary could be used to maintain a stable electrospray, contrary to the assumption that a positive charged coating with a strong electroosmotic flow (EOF) was required for a stable electrospray operation in a sheathless interface. Benefiting from minimized EOF by neutral coating, a broad separation window ( $\sim 0 \mathrm{~min})$ and a very high peak capacity ( 330) were achieved [22]. Higher sensitivity of the porous sheathless interface was demonstrated compared to a classical coaxial sheath liquid interface. Ramautar and co-workers further reported an 8-fold to 30-fold improvement in sensitivity by using the porous sheathless CE-ESI-MS as compared to a traditional sheath-liquid CE-ESI-MS, resulting in 3 times more molecular feature identification in human urine analysis [23]. The high peak capacity together with the high sensitivity made the porous, sheathless interface ideal for the analysis of very complex samples in minute amounts.

In an attempt to both increase the sample loading capacity and the sensitivity of CE-ESI-MS, a novel sheathless interface was proposed in our group by combining a large inner diameter (i.d.) separation capillary and a detachable small i.d. porous wall ESI emitter [24]. In this interface, as shown in Figure 5, the electrical contact at the emitter end of the CE separation capillary was realized by using the same porous sheathless interfaces. However, the new interface development allowed one end of the small i.d. emitter capillary to be inserted into 
the larger i.d. CE separation capillary. The capillary joint was sealed by carefully applying the acid resistant epoxy around it. The simultaneous achievement of large sample loading capacity and dilution free nanoESI operation significantly improved CE-ESI-MS sensitivity. A $10 \mathrm{pM}$ limit of quantification (LOQ) was obtained experimentally for targeted peptides in protein digest matrix, representing a 5-fold sensitivity improvement as compared to using the sheath liquid interface developed previously [25].

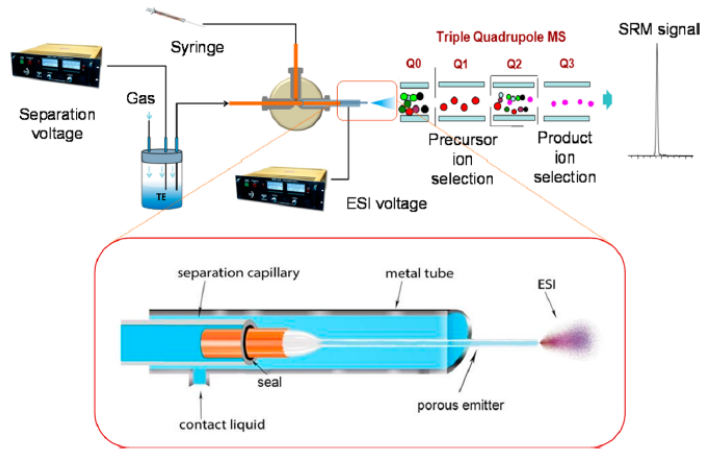

Figure 5 Schematic of the novel sheathless interface setup in our group. Reprinted with permission from reference [24]

Although the porous emitter sheathless interface is very effective in achieving high sensitivity CE-ESI-MS analysis, its fabrication process in the laboratory environment was found to be difficult to control. The porous emitter chemical etching process can be easily affected by minor variations of the etch solution concentration, total etching time, and the capillary dimension, making the reproducibility of the porous emitters problematic among different emitter batches which subsequently requires optimization of ESI voltages for the stable electrospray in each operation. Besides, the extremely thin porous wall also makes the emitter fragile to any minor perturbation during CE-ESI-MS sample analysis. To overcome the reproducibility and raggedness problems suffered by the porous emitter-based interfaces, a new sheathless interface was designed with merits of ease of fabrication and assembly, robust to CE-ESI-MS analysis, and good reproducibility while still maintaining overall high sensitivity of CE-ESI-MS [26]. These merits mainly resulted from two unique features of the interface. First, a single capillary was used as both the CE separation capillary and the ESI emitter capillary, where the ESI emitter end of the capillary was tapered by using either chemical etching or mechanical polishing and the outer surface of the tapered emitter was platinum-coated to provide electric conductivity. Second, the electrical contact between the ESI voltage power supply and the CE separation effluent was accomplished by using a conductive liquid that came in contact with the metal-coated outer surface of the emitter. The experimental setup of the sheathless interface is shown in Figure 6. The injection end of the separation capillary $(360 \mu \mathrm{m}$ o.d., $30 \mu \mathrm{m}$ i.d., and $1 \mathrm{~m}$ long) was inserted into a sealed liquid reservoir, where a platinum wire was inserted to provide CE separation voltage. The other end of the separation capillary was used as the ESI emitter. The majority of the platinumcoated surface of the emitter was further enclosed in a larger metal tube $(1.59 \mathrm{~mm}$ o.d., $1 \mathrm{~mm}$ i.d., and $30 \mathrm{~mm}$ long), filled with conductive liquid. The metal tube and the capillary were assembled together by using a metal tee. The top arm of the tee was connected to a syringe filled with conductive liquid so that the conductive liquid could be refreshed between sample analyses to prevent ion depletion in the solution and bubble accumulation generated from the electrochemical reaction at the metal surface. Once the ESI voltage was applied directly on the metal tube, the voltages needed by CE and ESI processes were built up through the metal tube contact, the conductive liquid, and the metal-coated surface of the emitter, making the interface a truly sheathless one. By using this indirect electrical contact through a conductive liquid, around one hundred analyses could be easily achieved without a noticeable loss of metal coating on the ESI emitter surface, or degrading of the ESI emitter performance, which demonstrated excellent robustness of the interface. The average correlation of variation (CV) at $0.8 \%$ for peak migration time from CE-ESI-MS analyses of a selected peptide standard at nine different concentrations also indicated an excellent reproducibility of this interface. A 5 attomole limit of quantification (LOQ) for Kemptide was achieved using this interface.

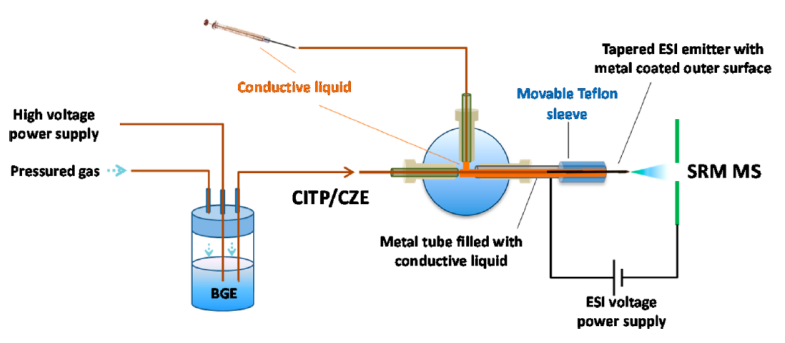

Figure 6 Schematic of the robust sheathless interface setup in our group. Reprinted with permission from reference [26]

\section{In-capillary sample preconcentration techniques}

Although CE-MS has the attractive features of high separation efficiency, fast analysis speed, and high sensitivity, it is still not considered as the mainstream 
method of choice in the analyses of biological samples and other complex mixtures as compared to well established LC-MS technique. One major reason is its limited loading capacity, while larger volume sample injections are often required for analyzing complex biological samples in order to effectively measure most biologically significant species typically existing at ultralow concentration [21]. With a total capillary volume of typically less than several microliters, CE is intrinsically a very small scale separation technique. As a rule of thumb, the sample loading capacity in a traditional capillary zone electrophoresis (CZE) separation is usually limited to about $1 \%$ of the total capillary volume in order to maintain a good separation quality, which limits the sample loading capacity to tens of nanoliter range. Taking a commonly used $30 \mu \mathrm{m}$ i.d., and $1 \mathrm{~m}$ long separation capillary as an example, the sample loading capacity for a good CZE separation would be only about $30 \mathrm{~nL}$. Alternative techniques aiming at increasing the sample loading capacity would be necessary in order to make the CE-based separation technique broadly applicable to routine biological sample analysis.

One of the techniques developed fairly recently is the transient capillary isotachophoresis/capillary zone electrophoresis (CITP/CZE, or t-ITP) technique, a hybrid CITP and CZE operation mode. This in-capillary sample preconcentration/separation technique is capable of achieving both large sample loading capacity and high efficient separation. The sample loading capacity of over $30 \%$ of the total separation capillary volume has been demonstrated by using transient CITP/CZE, increasing the sample loading volume from nanoliter range to several microliters [25]. In a transient CITP/ CZE, CITP sample preconcentration/focusing occurs at the initial stage, based on the ion mobility differences between sample analytes and the fast moving leading electrolytes, followed by CZE separation. The transient CITP/CZE offers the benefits of speed and straightforward manipulation/switching between the preconcentration (CITP) and separation (CZE) modes. In transient CITP/CZE, CITP is mainly used as a preconcentration method by which a concentration factor of three orders of magnitude can be achieved when applied to diluted samples. Furthermore, CITP can also selectively enrich low abundance analytes, since the concentration of the analytes after preconcentration is mainly determined by the concentration of the leading electrolyte according to the Kohlrausch equation [27]. Such selective enrichment capability of CITP toward low-abundance analytes can dramatically increase their intensities and therefore improve the concentration detection limit of targeted analytes. Busnel and co-workers demonstrated that using transient CITP/ CZE mode in a sheathless CE-ESI-MS setup improved the concentration detection limits of a digested E.coli culture by a factor ranging from 10 -fold to 86 -fold as compared to using CZE mode, leading to subnanomolar concentration detection limits [22]. An integrated transient CITP/CZE with a sheathless CE-ESI-MS for human urine analysis by Ramautar and co-workers also resulted in detection limit ranging from 0.6 to $24 \mathrm{nM}$, which were at least 5 -fold lower than the detection limit obtained by using CZE mode [23].

A potential drawback of transient CITP/CZE is the tradeoff between the gain in sample loading capacity and the loss in separation resolution at a given separation capillary dimension. As a large portion of separation capillary is used for the initial sample loading in transient CITP/CZE, a smaller portion of capillary is left for sample separation as compared with CZE only separation. This tradeoff has been optimized experimentally by Wang and co-workers [25]. In their study, base peak ion chromatograms (BPC) was measured under different sample loading volumes, controlled by varying sample loading time from 1.5 to $15 \mathrm{~min}$ (Figure 7). The peptide intensity continued to increase as the sample loading time increased from 1.5 to $7.5 \mathrm{~min}$ while the peak width remained essentially the same. Beyond the 10 min sample loading time, significant peak-broadening and separation resolution loss were observed. The area under the curve (AUC) of $\mathrm{BPC}$ versus loading time in Figure 7B also demonstrated the same trend. When the sample loading was increased beyond $10 \mathrm{~min}$, the rate of area increase (the slope of the curve) was decreased. This is largely due to the peak overlap resulting from insufficient separation time at large sample loading volume. Since the $7.5 \mathrm{~min}$ sample loading time provided the best balance between sample loading volume and separation resolution, it was selected as the optimum sample loading time, corresponding to $\sim 1 \mu \mathrm{L}$ sample loading volume or $25 \%$ of the total separation capillary volume. The migration times (MT) under different sample loading times, as shown in Figure $7 \mathrm{C}$, indicated that the MT increased initially and then decreased as sample loading time increased, indicating the dominant factor affecting MT changed from ion focusing time to ion separation time at the $7.5 \mathrm{~min}$ sample loading time. 


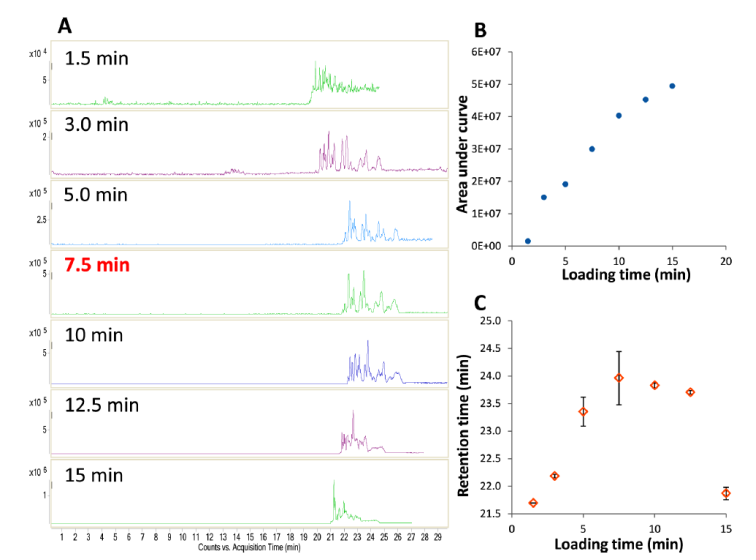

Figure 7 Sample loading capacity optimization. (A) BPC from CITP/CZE-ESI-MS with different sample loading time. (B) AUC of $B P C$ presented in panel $A$ with different sample loading times. (C) Migration time of peptide $m / z 432.3$ [VGTR + H] + . Sample conditions: $3.75 \mu \mathrm{M}$ BSA digest in $25 \mathrm{mM}$ ammonium acetate $(\mathrm{pH}$ $=4$ ). Loading condition: constant pressure at $1 \mathrm{psi}$. Reprinted with permission from reference [25]

Dynamic $\mathrm{pH}$ junction is another alternative in-capillary sample preconcentration technique which is ideally suited for amphiprotic analytes. The initial idea of dynamic $\mathrm{pH}$ junction was presented in the early 1990s, [28] and its application extends from amphiprotic analytes to weakly acidic compounds [29]. The mechanism of dynamic $\mathrm{pH}$ junction is based on a $\mathrm{pH}$ difference at the boundary between the sample and electrolyte, by which the amphiprotic analytes undergo rapid changes in their electrophoretic mobilities and focusing occurs at the $\mathrm{pH}$ boundary. In a typical $\mathrm{pH}$ junction operation, a plug of high-pH analyte sample are loaded into the capillary and two ends of the plug are surrounded by the low-pH BGE. Analytes bear a negative charge in the high-pH. Application of voltage induces the negatively charged analytes to migrate towards the anode. Once the analytes encounter the low-pH BGE, they acquire a positive charge, and their electrophoretic mobilities are reversed towards the cathode. In this way, the analytes become focused at the boundary between the high-pH sample solution and low-pH BGE. Once the plug of sample is neutralized by BGE, positively charged analytes start to migrate to the distal end of the capillary in a classical CZE separation mode. However, the precise mechanism of the dynamic $\mathrm{pH}$ junction may be quite complicated as CITP could be induced when different ions are used in the sample [30]. The dynamic $\mathrm{pH}$ junction can also be operated in a reverse mode, with a low-pH sample and high-pH BGE. Chen and coworkers investigated the capture efficiency of dynamic pH junction focusing by theoretical modeling. The simulation results demonstrated that both of the two dynamic $\mathrm{pH}$ junction modes can focus at least $95 \%$ of the target molecules injected into the capillary and a longer sample plug could capture a higher percentage of molecules [31]. Imami and coworkers investigated the focusing mechanism of the dynamic $\mathrm{pH}$ junction by using a pH boundary model. According to this mechanism, analytes dissolved in buffer with higher $\mathrm{pH}$ focused better when low-pH BGE was used. Their study also demonstrated the benefit of using dynamic $\mathrm{pH}$ junction in CE-ESI-MS for the qualitative identification of low-abundance proteins. Around 550-fold to 1000-fold sensitivity enhancement could be achieved in terms of peak intensity without degrading peak shape and resolution [32]. Zhu and coworkers found that using dynamic $\mathrm{pH}$ junction comparable protein and peptide identification was obtained by using $200 \mathrm{~nL}$ (1/6 of capillary volume) E. coli digest sample at 10 times lower sample concentration as compared to CZE only separation [33]. They also found the dynamic $\mathrm{pH}$ junction started to suffer separation window compression and resolution loss when the injection volumes were increased to half of capillary volume. One possible reason is the low quality of the neutral coating on the capillary inner wall used. Using a home-made high-quality neutral coating instead of commercial coated capillary in dynamic $\mathrm{pH}$ junction method, the Sun's group achieved still very high separation efficiency for proteins when 50\% of the capillary was filled with samples [34]. More recently, they even reported close to 1 million theoretical plates for CZE separation of proteins using the dynamic $\mathrm{pH}$ junction method [35].

\section{CE-ESI-MS for biological applications}

Benefited by the continued development of highly sensitive and robust CE-ESI-MS interfaces and highly efficient sample preconcentration techniques, CE-ESI-MS has been successfully applied to analysis of proteins, peptides, metabolites and glycan in the recent decade. Compared with LC-ESI-MS, CE-ESI-MS could yield higher sensitivity and therefore is capable of identifying more proteins and peptides when the sample amount is very limited. [17, 21, 36-38] A comparative proteomics measurements of E. coli protein digests indicated that $83 \%$ more peptides were identified by using an electro-kinetically driven sheath-flow CE coupled with a LTQ-Orbitrap Velos mass spectrometer as compared to using nano flow UPLC coupled with the same mass spectrometer when the sample loading amount was reduced to $1 \mathrm{ng}$. Although Zhu et.al reported a determination of reproducibility of CE-ESI-MS/MS in their paper, according to the description 
of experiment design, reproducibility of the method was tested [38]. Because of the high sensitivity and very small sample requirement in each CE-ESI-MS analysis, the technique is also applied effectively for single cell analysis. A metabolomics profiling by using a sheath liquid CE coupled with a Q-TOF MS demonstrated the detection of more than 100 compounds by injecting only $0.1 \%$ of the total cellular content from a single metacerebral cell extracted from Aplysia californica. Detection limits of the CE-ESI-MS instrument were shown to be in the low nanomolar range for a number of cell-to-cell signaling molecules, including acetylcholine (ACh), histamine, dopamine, and serotonin [39].

CE-ESI-MS has also been shown to becomplementary to LC-ESI-MS on peptides and proteins identifications (IDs) due to the different separation mechanisms between CE and LC separation. A combination of these two techniques in the protein enzymatic digest sample analysis can often improve the protein sequence coverage. A detailed proteomics measurement by using both CZE-ESI-MS/MS and UPLC-ESI-MS/MS on a tryptic digest of the secreted protein fraction from Mycobacterium marinum indicated that $62 \%$ of peptides identified from CZE-ESI-MS/MS analysis and 67\% from UPLC-ESI-MS/MS analysis were unique to each other. Combining the two data sets increased the total number of unique peptide identification by $53 \%$ (Figure 8). The similar complementary results in protein IDs were also obtained as shown in Figure 8. Their study also indicated that larger percentage of total peptide IDs identified by CZE-ESI-MS/MS had the mass-tocharge ratio $m / z<700$ as compared to by using UPLCESI-MS/MS (50\% vs 25\%), and peptides identified by CZE-ESI-MS/MS contained larger percentage of hydrophilic peptides as compared to UPLC-ESI-MS/ MS ( $55 \%$ vs $50 \%$ ), which were consistent with their corresponding separation mechanisms [40]. The main conclusions from this study were also consistent with an early study from Lindner's group. Among the total histone H1 peptides IDs from both CE-ESI-MS analysis of $300 \mathrm{fmol}$ sample and LC-ESI-MS analysis of $3 \mathrm{pmol}$ sample, $30.6 \%$ were identified solely by CE-ESI-MS and $27.4 \%$ were identified solely by LC-ESI-MS. The number of peptides identified could be increased by 44\% from 129 (LC-ESI-MS only) to 186 (LC-ESI-MS plus CE-ESI-MS). It was also found that CE-ESI-MS exhibited better identification of low molecular mass peptides (below $1400 \mathrm{Da}$ ) than LC-ESI-MS [21]. More recently, one deep bottom-up proteomics study showed the complementary characteristic between SCX-RPLCCZE-MS/MS and 2D-LC-MS/MS using very large datasets
(8200 vs. 8900 protein groups, 65,000 vs. 70,000 unique peptides). [14]

A

CZE-ESI-MS/MS nanoLC-ESI-MS/MS

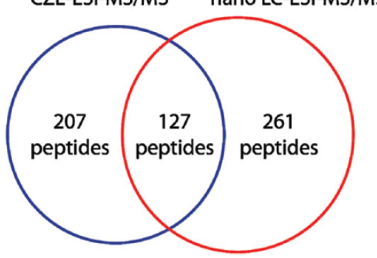

B

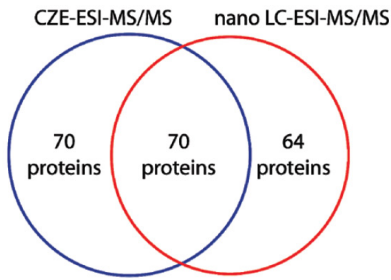

Figure 8 Venn diagram illustrating the overlap of peptides and proteins identified by both CZE-ESI-MS/MS and UPLC-ESI-MS/MS:

(A) peptides, (B) proteins. Reprinted with permission from reference [40]

CE and LC separation can also be combined for two dimensional separation due to their different separation mechanisms, in order to gain a combined separation peak capacity. A recent study by Nemes group illustrated this concept in which the complex cell digest sample was first fractionated by the reversed phase LC (RPLC) separation followed by the CE-ESI-MS analysis of each sample fraction [41]. Specifically in their study, an $\sim 1$ to $20 \mu \mathrm{g}$ neuronal protein digest was first fractionated on a RPLC column, and $1 \mathrm{ng}$ to $500 \mathrm{pg}$ of peptides were analyzed by the CE-high resolution MS (HRMS). From 1 ng of protein digest ( $<5$ neurons), CE-HRMS with RPLC fractionation identified 737 proteins from 1,753 peptide IDs. The approach was also shown to be scalable to $500 \mathrm{pg}$ of protein digest, identifying 225 proteins from 623 peptide IDs in technical triplicate analyses. Among identified proteins, 101 proteins were products of genes that are known to be transcriptionally active in single neurons during early development of the brain, including those involved in synaptic transmission and plasticity and cytoskeletal organization. The study demonstrated that a combined LC fractionation with CE-HRMS can push the sensitivity of the technique essentially to a few neuron level. Nemes group also did several noticeable works on single-cell proteomics using home-customed CE- $\mu$ ESI-HRMS platform and protocol [42, 43]. The Sun group did several works recently about coupling nanoflow RPLC to CZE-MS/MS for ultrasensitive proteomics, identifying 6500 proteins from a MCF7 proteome digest starting with only $500 \mathrm{ng}$ of peptides [44, 45]. They also reported the identification and quantification of thousands of proteoforms from zebrafish brains using CZE-MS/MS-based top-down proteomics with the consumption of nanograms of proteins [46].

Because of the extreme sensitivity of CE-ESI-MS, the technique is also very effective in quantifying targeted 
peptides/proteins in complex biological matrix, making it an effective tool for biomarker verification and validation. A recent study in our group that coupling CE with a triple quadrupole MS operating in selected reaction monitoring (SRM) operation mode can be very effective in quantifying low abundant peptide targets in complex bio-matrix. The demonstrative study by using peptide standard spiked into the tryptic digest of bovine serum albumin matrix at different concentrations demonstrated a 5 attomole limit of quantification (LOQ) and over 4 orders of magnitude of linear dynamic range [26]. Similar results were also obtained by the measurements from other groups. For example, a $60 \mathrm{pM}$ limit of detection, which corresponds to the injection of $335 \mathrm{zmol}$ of peptide, was achieved using electro-kinetically driven sheath-flow interface combining with SRM-MS by using Leu-enkephalin spiked into the tryptic digest of bovine serum albumin matrix representing a 10-20 fold improvement in sensitivity as compared to using nano flow HPLC/SRM-MS for the same sample analysis [47]. In a subsequent study by using an improved CE interface from the same group, the sensitivity of CE-ESI-MS was drastically improved to be estimated at $\sim 1 \mathrm{zmol}$ peptide detection limit $(\mathrm{S} / \mathrm{N}=3)$ from $400 \mathrm{fg}$ E.coli digest, [18]. which pushed CE-MS sensitivity essentially to a single cell level.

\section{Closing Remarks}

CE-ESI-MS is a promising alternative technique to LC-MS in identifying and quantifying trace amounts of analytes in complex bio-matrixes due to its high separation efficiency and high detection sensitivity. It is expected that as CE-ESI-MS technique continues to evolve, even higher sensitivity will be achieved together with a better reproducibility making it a promising tool for single cell analysis. The significant improvement in the ruggedness of the CE-ESI-MS would also make the technique a routine analytical tool broadly applicable to many new chemical and biological applications.

\section{Acknowledgments}

This work was partially supported by the K. C. Wong Magna Fund in Ningbo University.

\section{Conflict of Interest}

The authors declare no conflict of interest.

\section{References}

[1] Shi T, Su D, Liu T, Tang K, Camp DG, Qian WJ, et al. Advancing the sensitivity of selected reaction monitoring-based targeted quantitative proteomics. Proteomics. 2012;12(8):1074-92.

[2] Klepárník K. Recent advances in combination of capillary electrophoresis with mass spectrometry: methodology and theory. Electrophoresis. 2015;36(1):159-78.

[3] Olivares JA, Nguyen NT, Yonker CR, Smith RD. On-line mass spectrometric detection for capillary zone electrophoresis. Anal Chem. 1987;59(8):1230-2.

[4] Heemskerk AA, Deelder AM, Mayboroda OA. CE-ESI-MS for bottom-up proteomics: advances in separation, interfacing and applications. Mass Spectrom Rev. 2016;35(2):259-71.

[5] Breadmore MC, Wuethrich A, Li F, Phung SC, Kalsoom U, Cabot JM, et al. Recent advances in enhancing the sensitivity of electrophoresis and electrochromatography in capillaries and microchips (2014-2016). Electrophoresis. 2017;38(1):33-59.

[6] Stolz A, Jooß K, Höcker O, Römer J, Schlecht J, Neusüß C. Recent advances in capillary electrophoresis-mass spectrometry: Instrumentation, methodology and applications. Electrophoresis. 2019;40(1):79-112.

[7] De Jong G. Capillary Electrophoresis-Mass Spectrometry (CE-MS): Principles and Applications. John Wiley \& Sons; 2016.

[8] Batz NG, Mellors JS, Alarie JP, Ramsey JM. Chemical Vapor Deposition of Aminopropyl Silanes in Microfluidic Channels for Highly Efficient Microchip Capillary ElectrophoresisElectrospray Ionization-Mass Spectrometry. Anal Chem. 2014;86(7):3493-500.

[9] Gilliland WM, Mellors JS, Ramsey JM. Coupling Microchip Electrospray Ionization Devices with High Pressure Mass Spectrometry. Anal Chem. 2017;89(24):13320-5.

[10] Zhang Z, Qu Y, Dovichi NJ. Capillary zone electrophoresis-mass spectrometry for bottom-up proteomics. Trends Analyt Chem. 2018;108:23-37.

[11] Gomes FP, Yates JR. Recent trends of capillary electrophoresismass spectrometry in proteomics research. Mass Spectrom Rev. 2019;38(6):445-60.

[12] Shen X, Yang Z, McCool EN, Lubeckyj RA, Chen D, Sun L. Capillary zone electrophoresis-mass spectrometry for top-down proteomics. Trends Analyt Chem. 2019;120:115644.

[13] McCool EN, Lubeckyj RA, Shen X, Chen D, Kou Q, Liu X, et al. Deep Top-Down Proteomics Using Capillary Zone Electrophoresis-Tandem Mass Spectrometry: Identification of 5700 Proteoforms from the Escherichia coli Proteome. Anal Chem. 2018;90(9):5529-33.

[14] Chen D, Shen X, Sun L. Strong cation exchange-reversed phase liquid chromatography-capillary zone electrophoresistandem mass spectrometry platform with high peak capacity for deep bottom-up proteomics. Anal Chim Acta. 2018;1012:1-9.

[15] Smith RD, Olivares JA, Nguyen NT, Udseth HR. Capillary zone electrophoresis-mass spectrometry using an electrospray ionization interface. Anal Chem. 1988;60(5):436-41.

[16] Maxwell EJ, Zhong X, Zhang H, van Zeijl N, Chen DD. Decoupling CE and ESI for a more robust interface with MS. Electrophoresis. 2010;31(7):1130-7. 
[17] Sun L, Zhu G, Zhang Z, Mou S, Dovichi NJ. Third-generation electrokinetically pumped sheath-flow nanospray interface with improved stability and sensitivity for automated capillary zone electrophoresis-mass spectrometry analysis of complex proteome digests. J Proteome Res. 2015;14(5):2312-21.

[18] Sun L, Zhu G, Zhao Y, Yan X, Mou S, Dovichi NJ. Ultrasensitive and fast bottom-up analysis of femtogram amounts of complex proteome digests. Angew Chem Int Ed. 2013;52(51):13661-4.

[19] Maxwell EJ, Chen DD. Twenty years of interface development for capillary electrophoresis-electrospray ionization-mass spectrometry. Anal Chim Acta. 2008;627(1):25-33.

[20] Moini M, Simplifying CE. MS operation. 2. Interfacing low-flow separation techniques to mass spectrometry using a porous tip. Anal Chem. 2007;79(11):4241-6.

[21] Faserl K, Sarg B, Kremser L, Lindner H. Optimization and evaluation of a sheathless capillary electrophoresiselectrospray ionization mass spectrometry platform for peptide analysis: comparison to liquid chromatographyelectrospray ionization mass spectrometry. Anal Chem. 2011;83(19):7297-305.

[22] Busnel JM, Schoenmaker B, Ramautar R, Carrasco-Pancorbo A, Ratnayake C, Feitelson JS, et al. High capacity capillary electrophoresis-electrospray ionization mass spectrometry: coupling a porous sheathless interface with transientisotachophoresis. Anal Chem. 2010;82(22):9476-83.

[23] Ramautar R, Busnel JM, Deelder AM, Mayboroda OA. Enhancing the coverage of the urinary metabolome by sheathless capillary electrophoresis-mass spectrometry. Anal Chem. 2011;84(2):885-92.

[24] Wang C, Lee CS, Smith RD, Tang K. Capillary isotachophoresisnanoelectrospray ionization-selected reaction monitoring MS via a novel sheathless interface for high sensitivity sample quantification. Anal Chem. 2013;85(15):7308-15.

[25] Wang C, Lee CS, Smith RD, Tang K. Ultrasensitive sample quantitation via selected reaction monitoring using CITP/CZE-ESI-triple quadrupole MS. Anal Chem. 2012;84(23):10395-403.

[26] Guo X, Fillmore TL, Gao Y, Tang K. Capillary ElectrophoresisNanoelectrospray Ionization-Selected Reaction Monitoring Mass Spectrometry via a True Sheathless Metal-Coated Emitter Interface for Robust and High-Sensitivity Sample Quantification. Anal Chem. 2016;88(8):4418-25.

[27] Stegehuis D, Irthu H, Tjaden U, Van der Greef J. Isotachophoresis as an on-line concentration pretreatment technique in capillary electrophoresis. J Chromatogr A. 1991;538(2):393-402.

[28] Aebersold R, Morrison HD. Analysis of dilute peptide samples by capillary zone electrophoresis. J Chromatogr A. 1990;516(1):79-88.

[29] Britz-McKibbin P, Chen DD. Selective focusing of catecholamines and weakly acidic compounds by capillary electrophoresis using a dynamic $\mathrm{pH}$ junction. Anal Chem. 2000;72(6):1242-52.

[30] Kazarian AA, Hilder EF, Breadmore MC. Online sample pre-concentration via dynamic $\mathrm{pH}$ junction in capillary and microchip electrophoresis. J Sep Sci. 2011;34(20):2800-21.

[31] Wang L, MacDonald D, Huang X, Chen DD. Capture efficiency of dynamic $\mathrm{pH}$ junction focusing in capillary electrophoresis. Electrophoresis. 2016;37(9):1143-50.
[32] Imami K, Monton MR, Ishihama Y, Terabe S. Simple on-line sample preconcentration technique for peptides based on dynamic $\mathrm{pH}$ junction in capillary electrophoresis-mass spectrometry. J Chromatogr A. 2007;1148(2):250-5.

[33] Zhu G, Sun L, Yan X, Dovichi NJ. Bottom-up proteomics of Escherichia coli using dynamic $\mathrm{pH}$ junction preconcentration and capillary zone electrophoresis-electrospray ionizationtandem mass spectrometry. Anal Chem. 2014;86(13):6331-6.

[34] Lubeckyj RA, McCool EN, Shen X, Kou Q, Liu X, Sun L. Single-Shot Top-Down Proteomics with Capillary Zone Electrophoresis-Electrospray Ionization-Tandem Mass Spectrometry for Identification of Nearly 600 Escherichia coli Proteoforms. Anal Chem. 2017;89(22):12059-67.

[35] Lubeckyj RA, Basharat AR, Shen X, Liu X, Sun L. Large-Scale Qualitative and Quantitative Top-Down Proteomics Using Capillary Zone Electrophoresis-Electrospray IonizationTandem Mass Spectrometry with Nanograms of Proteome Samples. J Am Soc Mass Spectrom. 2019;30(8):1435-45.

[36] Wang Y, Fonslow BR, Wong CC, Nakorchevsky A, Yates II Jr. Improving the comprehensiveness and sensitivity of sheathless capillary electrophoresis-tandem mass spectrometry for proteomic analysis. Anal Chem. 2012;84(20):8505-13.

[37] Chen D, Shen X, Sun L. Capillary zone electrophoresis-mass spectrometry with microliter-scale loading capacity, $140 \mathrm{~min}$ separation window and high peak capacity for bottom-up proteomics. Analyst (Lond). 2017;142:2118-27.

[38] Zhu G, Sun L, Yan X, Dovichi NJ. Single-Shot Proteomics Using Capillary Zone Electrophoresis-Electrospray IonizationTandem Mass Spectrometry with Production of More than 1250 Escherichia coli Peptide Identifications in a $50 \mathrm{~min}$ Separation. Anal Chem. 2013;85(5):2569-73.

[39] Lapainis T, Rubakhin SS, Sweedler JV. Capillary electrophoresis with electrospray ionization mass spectrometric detection for single-cell metabolomics. Anal Chem. 2009;81(14):5858-64.

[40] Li Y, Champion MM, Sun L, Champion PA, Wojcik R, Dovichi NJ. Capillary zone electrophoresis-electrospray ionizationtandem mass spectrometry as an alternative proteomics platform to ultraperformance liquid chromatographyelectrospray ionization-tandem mass spectrometry for samples of intermediate complexity. Anal Chem. 2012;84(3):1617-22.

[41] Choi SB, Lombard-Banek C. Muñoz-LLancao P, Manzini MC, Nemes P. Enhanced Peptide Detection Toward Single-Neuron Proteomics by Reversed-Phase Fractionation Capillary Electrophoresis Mass Spectrometry. J Am Soc Mass Spectrom. 2017;1-10.

[42] Lombard-Banek C, Moody SA, Nemes P. Single-Cell Mass Spectrometry for Discovery Proteomics: Quantifying Translational Cell Heterogeneity in the 16-Cell Frog (Xenopus) Embryo. Angew Chem Int Ed. 2016;55(7):2454-8.

[43] Onjiko RM, Portero EP, Moody SA, Nemes P. In Situ Microprobe Single-Cell Capillary Electrophoresis Mass Spectrometry: Metabolic Reorganization in Single Differentiating Cells in the Live Vertebrate (Xenopus laevis) Embryo. Anal Chem. 2017;89(13):7069-76.

[44] Yang Z, Shen X, Chen D, Sun L. Microscale Reversed-Phase Liquid Chromatography / Capillary Zone ElectrophoresisTandem Mass Spectrometry for Deep and Highly Sensitive 
Bottom-Up Proteomics: Identification of 7500 Proteins with Five Micrograms of an MCF7 Proteome Digest. Anal Chem. 2018;90(17):10479-86.

[45] Yang Z, Shen X, Chen D, Sun L. Improved Nanoflow RPLC-CZE-MS/MS System with High Peak Capacity and Sensitivity for Nanogram Bottom-up Proteomics. J Proteome Res. 2019;18(11):4046-54.

[46] Lubeckyj RA, Basharat AR, Shen X, Liu X, Sun L. Large-Scale Qualitative and Quantitative Top-Down Proteomics Using
Capillary Zone Electrophoresis-Electrospray IonizationTandem Mass Spectrometry with Nanograms of Proteome Samples. J Am Soc Mass Spectrom. 2019;30(8):1435-45.

[47] Li Y, Wojcik R, Dovichi NJ, Champion MM. Quantitative multiple reaction monitoring of peptide abundance introduced via a capillary zone electrophoresis-electrospray interface. Anal Chem. 2012;84(14):6116-21. 